\title{
Solitons and oscillitons in multi-ion space plasmas
}

\author{
K. Sauer ${ }^{1}$, E. Dubinin ${ }^{1}$, and J. F. McKenzie ${ }^{1,2}$ \\ ${ }^{1}$ Max-Planck Institut für Aeronomie, Katlenburg-Lindau, Germany \\ ${ }^{2}$ School of Pure and Applied Physics, University of Natal, Durban, South Africa
}

Received: 25 June 2001 - Revised: 25 March 2002 - Accepted: 9 April 2002

\begin{abstract}
It is well known that additional low-frequency waves arise when a second ion population is added to a plasma normally consisting of protons and electrons. Here, we investigate stationary structures streaming with a sub-fast speed in such a bi-ion plasma. It is shown that in addition to the usual "solitons", which have already been described for a single-ion plasma, a new type of stationary structure occurs due to the second ion population. This structure is associated with complex solutions of the linear dispersion relation in certain regions of the wave number-obliquity space. This implies that the corresponding soliton structure exhibits an oscillating spatial structure superposed on the usual spatial growth or decay. The full-blown solution of the nonlinear equations confirms that this is indeed the case. The related structure is called an "oscilliton". Examples of both types of stationary nonlinear waves (solitons and oscillitons), which may exist in a bi-ion plasma are given.
\end{abstract}

\section{Introduction}

In many space plasma phenomena heavy ions are present in abundance which is not negligible relative to the protons. The solar wind contains $\alpha$-particles and other minor heavy ions. Near nonmagnetized planets, such as Venus or Mars, where the solar wind interacts directly with planetary ionospheres (exospheres), the incoming solar wind plasma is also "contaminated" by heavy ions of planetary origin. Comets provide another clear example where the solar wind is massloaded by cometary ions originating from the neutral gas envelope around the comet. The traditional view that magnetospheric plasmas consist mainly of plasma of solar wind origin is now challenged (see e.g. Chappel et al., 1987). The ionospheres and plasmaspheres of magnetized planets also provide a very effective source for magnetospheric plasmas. Planetary moons, such as Io or Titan, supply the planetary

Correspondence to: K. Sauer (sauer@linmpi.mpg.de) magnetospheres with neutral gas which is ionized and contributes significantly to the plasma content.

Space observations near celestial bodies with a multi-ion plasma environment provide many examples of large-amplitude coherent low-frequency waves. However, their generation mechanism is still unclear. In most cases, the waves were attributed to beam-like instabilities excited by pickedup secondary ions, although the question of why the observed waves are so coherent remains open. In this paper, we investigate stationary nonlinear structures in a homogeneous, equilibrium plasma containing two ion populations using multi-fluid equations. The concept of a multi-fluid description is very useful and has been applied to solar wind mass loading at unmagnetized planets and comets (see e.g. Sauer et al., 1994, 1996, 1997; Dubinin and Sauer, 1999). Stationary waves in a bi-ion plasma, propagating transverse to the magnetic field, were discussed recently by Sauer et al. (2000), McKenzie et al. (2001), Dubinin et al. (2002). The study is based on structure equations of the system that are derived from the equations of motion and Maxwell equations. They describe the electromagnetic coupling between the two ion fluids and the electrons through the Lorentz forces and the charge neutrality constraint. Soliton solutions have been found for a specific range of flow velocities. The structure of compressive solitons depends upon the initial Mach number, the abundance ratio of the heavy ions and the mass ratio.

In this paper, we analyse obliquely propagating stationary waves in bi-ion plasmas. Certain types of solitons in biion plasma have also been studied by Verheest (1990) and Hackenberg et al. (1998). Verheest (1990) found soliton solutions for Alfvén waves propagating parallel to the magnetic field when the ion species stream differentially. The structure equation in this case can be reduced to the deriative nonlinear Schrodinger type. Hackenberg et al. (1998) considered oblique solitons in a multi-fluid approach, but was restricted to very particular cases which give rise to the usual solitons.

The layout of the paper is as follows. Hall MHD solitons in a single-ion plasma are presented in Sect. 2. These solu- 
tions are modified by the addition of a second ion population which leads to the appearance of solitons with an oscillating substructure, called "oscillitons" (s.a. Sauer et al., 2001). In Sect. 3, the properties of "oscillitons" in a bi-ion plasma propagating obliquely to the magnetic field are considered. Applications to space observations are discussed.

\section{Hall-MHD solitons in a single-ion plasma}

In this section, we discuss briefly the structure of one-dimensional solitons propagating in a single-ion plasma obliquely to the magnetic field. Hall MHD equations are used, which adequately describe the plasma behaviour in the lowfrequency range. Subsequently, the same method is applied to plasmas consisting of two-ion populations.

The standard multi-fluid equations for protons (p) and electrons (e) are

$\frac{\partial n_{i}}{\partial t}+\nabla n_{i} \mathbf{v}_{i}=0$

$m_{i} n_{i} \frac{D_{i} \mathbf{v}_{i}}{D t}+\nabla p_{i}=e n_{i} q_{i}\left(\mathbf{E}+\mathbf{v}_{i} \times \mathbf{B}\right)$

where $m_{i}$ is the mass, $q_{i}$ the ion charge, $n_{i}$ the density, $\mathbf{v}_{i}$ the velocity of each species $(i=p, e), \mathbf{E}$ and $\mathbf{B}$ are the electric and magnetic fields, respectively. The convective derivative is

$$
\frac{D_{i}}{D t}=\frac{\partial}{\partial t}+\mathbf{v}_{i} \cdot \nabla \text {. }
$$

The electric and magnetic field, respectively, follows from Faraday's law

$$
\frac{\partial \mathbf{B}}{\partial t}+\nabla \times \mathbf{E}=0
$$

and Ampere's law (neglecting the displacement current)

$$
\nabla \times \mathbf{B}=\mu_{0} \mathbf{j},
$$

where the current $\mathbf{j}$ is given by

$j=e\left(n_{p} \mathbf{v}_{p}-n_{e} \mathbf{v}_{e}\right)$.

Using the approximation of massless electrons, $m_{e} \rightarrow 0$, the electric field can be eliminated from Eq. (2) as

$\mathbf{E}=-\mathbf{v}_{e} \times \mathbf{B}-\frac{1}{n_{e}} \nabla p_{e}$.

Thus, the equation of motion for the protons becomes

$$
\frac{\partial}{\partial t} n_{p} \mathbf{v}_{p}+\nabla \cdot n_{p} \mathbf{v}_{p} \mathbf{v}_{p}+\frac{1}{m_{p}} \nabla\left(p_{p}+p_{e}\right)=\frac{e}{m_{p}}\left(\mathbf{v}_{p}-\mathbf{v}_{e}\right) \times \mathbf{B},
$$

where the quasi-neutrality condition $n_{p}=n_{e}$ has been used.

For the one-dimensional case in which all parameters depend only on the $x$-coordinate and the undistubed magnetic field lies in the $x-z$ plane, the corresponding set of equations describing nonlinear stationary structures can be written as follows, continuity equation:

$$
\frac{d}{d x}\left(n_{i} v_{i x}\right)=0,(i=p, e)
$$

equations of motion:

$$
\begin{aligned}
& m_{p} v_{p x} \frac{d v_{p x}}{d x}=e\left(v_{p y}-v_{e y}\right) B_{z} \\
&-e\left(v_{p z}-v_{e z}\right) B_{y}-\frac{1}{n_{p}} \frac{d p_{e}}{d x} \\
& m_{p} v_{p x} \frac{d v_{p y}}{d x}=e\left[-\left(v_{p x}-v_{e x}\right) B_{z}+\left(v_{p z}-v_{e z}\right) B_{x}\right] \\
& m_{p} v_{p x} \frac{d v_{p z}}{d x}=e\left[\left(v_{p x}-v_{e x}\right) B_{y}-\left(v_{p y}-v_{e y}\right) B_{x}\right]
\end{aligned}
$$

Faraday's law:

$$
\begin{aligned}
& \frac{d}{d x}\left(v_{e x} B_{y}\right)-B_{x} \frac{d}{d x} v_{e y}=0 \\
& \frac{d}{d x}\left(v_{e x} B_{z}\right)-B_{x} \frac{d}{d x} v_{e z}=0
\end{aligned}
$$

Ampere's law:

$\frac{d B_{y}}{d x}=-\mu_{0} e n_{p}\left(v_{e z}-v_{p z}\right)$

$\frac{d B_{z}}{d x}=+\mu_{0} e n_{p}\left(v_{e y}-v_{p y}\right)$

In a frame moving with a speed $U$ one can search for stationary structures by putting $v_{p x} \rightarrow v_{p x}-U$. Then, with the boundary conditions $n_{p}=n_{p o}, v_{p x}=0$, the continuity equation for the protons becomes

$n_{p}\left(U-v_{p x}\right)=n_{p o} U$,

where $n_{p o}$ is the equilibrium density.

From Faraday's law (11), one can express the $y$ and $z$ components of the electron velocity as

$v_{e y}=-\frac{U n_{p o}}{n_{p}} \frac{B_{y}}{B_{x}}$

$v_{e z}=-\frac{U}{B_{x}}\left(\frac{n_{p o}}{n_{p}} B_{z}-B_{z o}\right)$,

where

$v_{e x}=v_{p x}=U\left(1-\frac{n_{p o}}{n_{p}}\right)$

is used which follows from the condition $j_{x}=e\left(n_{p} v_{p x}-\right.$ $\left.n_{e} v_{e x}\right)=0$ and $n_{p}=n_{e}$. Combining the proton momentum Eqs. (10) and Ampere's law (12), one obtains the following expressions for the proton velocities

$v_{p x}=\frac{1}{2 n_{p o} m_{p} U}\left[\frac{B^{2}-B_{0}^{2}}{\mu_{0}}+2\left(p_{e}-p_{e o}\right)\right]$ 
$v_{p y}=-\frac{B_{x} B_{y}}{\mu_{0} m_{p} n_{p o} U}$

$v_{p z}=-\frac{B_{x}\left(B_{z}-B_{z o}\right)}{\mu_{0} m_{p} n_{p o} U}$,

where $B^{2}=B_{x}^{2}+B_{y}^{2}, B_{z}^{2}$ and $B_{0}^{2}=B_{x o}^{2}+B_{z o}^{2}, B_{x}=B_{x o}$.

Assuming isothermal electrons $\left(\gamma_{e}=1\right), p_{e}=$ $p_{e o} n_{p} / n_{p o}$, the proton velocity $v_{e x}$ is according to Eq. (15a), determined by a quadratic equation.

For practical purposes, dimensionless quantities are introduced by normalizing the velocities to the proton Alfvén speed $V_{A p}=B_{0} /\left(\mu_{0} n_{p o} m_{p}\right)^{1 / 2}$ and the magnetic field to the undisturbed value $B_{0}$. The ordinary differential equations for the wave magnetic field can be written as

$$
\begin{gathered}
\frac{d B_{y}}{d x}=\frac{1}{\left(U-v_{p x}\right) B_{x}} \\
{\left[\left(U^{2}-B_{x o}^{2}\right)\left(B_{z}-B_{z o}\right)-U v_{p x} B_{z}\right],} \\
\frac{d B_{z}}{d x}=\frac{B_{y}}{\left(U-v_{p x}\right) B_{x}}\left[U^{2}-B_{x o}^{2}-U v_{p x}\right],
\end{gathered}
$$

where $v_{p x}$ follows from the quadratic Eq. (15a)

$v_{p x}^{2}-P v_{p x}+Q=0$

with

$P=\left[U^{2}-\beta_{e}+\frac{1}{2}\left(B^{2}-1\right)\right] / U$,

$Q=\frac{1}{2}\left(B^{2}-1\right)$,

and

$\beta_{e}=\frac{p_{e o}}{B_{0}^{2} / 2 \mu_{0}}$

The spatial coordinate $x$ is normalized with respect to the proton inertial length $L=V_{A p} / \Omega_{p}=c / \omega_{p}\left(\omega_{p}\right.$ : proton plasma frequency). The other (normalized) velocity components are given by

$v_{p y}=-\frac{B_{x} B_{z}}{U}$

$v_{p z}=-\frac{B_{x}}{U}\left(B_{z}-B_{z o}\right)$.

The coupled, nonlinear differential Eq. (16) for the magnetic field, together with Eq. (17) for $v_{p x}$, determine the spatial structure of stationary waves in a single-ion plasma. Their linearization in the neighbourhood of the initial state yields stationary waves which may be either of the evanescent or sinusoidal type.

The right panels in Fig. 1 show the real and imaginary parts of $k$ as a function of the (soliton) velocity $U$ for $\theta=80^{\circ}$ and $\beta_{e}=3$. For a single-ion plasma with finite electron temperature, there are generally two gaps in which $k^{2}<0$, indicating evanescent solutions. Here, only the gap between
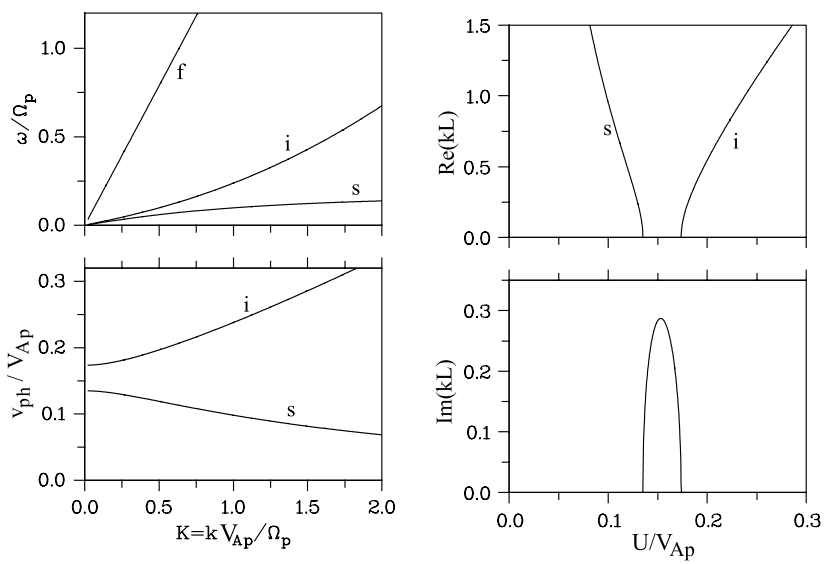

Fig. 1. (left) Dispersion curves of low-frequency waves in a singleion plasma (Hall-MHD approach) for $\theta=80^{\circ}, \beta_{e}=3$. The frequency is normalized to the proton gyrofrequency. (right) Dispersion of stationary waves $k=k(U)$, where the soliton velocity $U$ is normalized to the Alfvén speed. $L$ is the proton inertial length, $L=V_{A p} / \Omega_{p}$. Evanescent solutions exist in the gap between the slow $(s)$ and intermediate mode $(i)$.

the slow (s) and intermediate (i) near $U \sim 0.15$ is shown. There is also a gap between the fast (f) and intermediate mode where soliton-like solutions can exist. The dispersion curves for propagating waves shown in the left panel of Fig. 1 are readily found by using the tranformation $U \rightarrow \omega / k$.

Figure 2 shows two examples of compression and rarefaction solitons (so-called "dark" and "bright" solitons) propagating with small velocities at large angles to the magnetic field.

Baumgärtel et al. (1997) and Baumgärtel (1999) have considered compression solitons in more detail and applied these solutions to magnetic holes often observed in space plasmas (Winterhalter et al., 1994). McKenzie and Doyle (2001a,b) have carried out an exact analysis of oblique solitons in both cold and hot magnetized plasmas and described their properties.

\section{Bi-ion solitons and oscillitons}

In this section, we focus on new features which appear when a second ion population is added to the main plasma. In many space plasmas, heavy ions are present in abundance which is not negligible relative to the protons. The solar wind plasma is composed of protons and alpha particles. Cometary plasmas are generally a mixture of the solar wind that is dominated by protons and cometary matter which becomes ionized by photoionization and charge-exchange processes. Multi-ion plasmas also exist near nonmagnetized planets where the solar wind has direct access to ionospheric and atmospheric planetary shells.

We consider a plasma with two ion species and treat protons and heavy ions as separate cold $\left(T_{p}=T_{h}=0\right)$ fluids. The abundance and mass ratio are $\alpha=n_{h o} / n_{p o}$ and $\mu=m_{h} / m_{p}$, respectively. The derivation of the coupled set 
of ordinary differential equations describing stationary waves is similar to that for single-ion plasmas given in the previous section.

First, the momentum equation for the protons is considered. In normalized units the equations for the $x$ and $y$ velocity components can be written as

$\left(v_{p x}-U\right) \frac{d v_{p y}}{d x}=\frac{q_{p}}{\mu_{p}}\left[E_{y}+v_{p z} B_{x}-v_{p x} B_{z}\right]$

$\left(v_{p x}-U\right) \frac{d v_{p z}}{d x}=\frac{q_{p}}{\mu_{p}}\left[E_{z}-v_{p y} B_{x}+v_{p x} B_{z}\right]$.

(In the following, normalized quantities are used throughout.)

With the relations for the electric field

$E_{y}=-U B_{z o}$,

$E_{z}=0$

and the continuity equation, one finds

$\frac{d v_{p y}}{d x}=-\frac{q_{p}}{\mu_{p}}\left[B_{z}+\frac{n_{p} v_{p z} B_{x}}{U}-n_{p} B_{z o}\right]$

$\frac{d v_{p z}}{d x}=+\frac{q_{p}}{\mu_{p}}\left[B_{y}+\frac{n_{p} v_{p y} B_{x}}{U}\right]$.

Here, $q_{p}=1$ and $\mu_{p}=1$. Similar equations hold for the heavy ions by replacing $p$ with $h$ to give

$\frac{d v_{h y}}{d x}=-\frac{q_{h}}{\mu_{h}}\left[B_{z}+\frac{n_{h} v_{h z} B_{x}}{U}-n_{h} B_{z o}\right]$

$\frac{d v_{h z}}{d x}=+\frac{q_{h}}{\mu_{h}}\left[B_{y}+\frac{n_{h} v_{h y} B_{x}}{U}\right], \mu_{h}=\mu=\frac{m_{h}}{m_{p}}$.

Two of the differential equations above can be replaced by algebraic relations using the overall momentum conservation of a bi-ion plasma in the $y$ and $z$ directions, which yields the expressions

$v_{p y}=-\alpha \mu v_{h y}-\frac{B_{x} B_{y}}{U}$

$v_{p z}=-\alpha \mu v_{h z}-\frac{B_{x}\left(B_{z}-B_{z o}\right)}{U}$.

Ampere's law becomes

$\frac{d B_{y}}{d x}=+\left(-n_{e} v_{e z}+n_{p} v_{p z}+n_{h} v_{h z}\right)$,

$\frac{d B_{z}}{d x}=-\left(-n_{e} v_{e y}+n_{p} v_{p y}+n_{h} v_{h y}\right)$,

where the electron velocities can be written as

$$
\begin{aligned}
& v_{e y}=-U \frac{(1+\alpha)}{n_{e}} \frac{B_{y}}{B_{x}} \\
& v_{e z}=-\frac{U}{B_{x}}\left[\frac{(1+\alpha)}{n_{e}} B_{z}-B_{z o}\right] .
\end{aligned}
$$

Here, the heavy ions are assumed to be single-ionized species $\left(q_{p}=q_{h}=1\right)$.

The remaining quantities to be determined are the number densities of protons and heavy ions, $n_{p}$ and $n_{h}$, or the related velocities $v_{p x}$ and $v_{h x}$. The corresponding equations of motion are

$\left(v_{p x}-U\right) \frac{d v_{p x}}{d x}=\frac{q_{p}}{\mu_{p}}\left[E_{x}+v_{p y} B_{z}-v_{p z} B_{y}\right]$

$\left(v_{h x}-U\right) \frac{d v_{p x}}{d x}=\frac{q_{h}}{\mu_{h}}\left[E_{x}+v_{h y} B_{z}-v_{h z} B_{y}\right]$,

where

$E_{x}=-\left(v_{e y} B_{z}-v_{e z} B_{y}\right)-0.5 \frac{\beta_{e}}{n_{e}} \frac{d n_{e}}{d x}$

and

$\left(v_{p x}-U\right) n_{p}=-U$,

$\left(v_{h x}-U\right) n_{h}=-\alpha U$,

$n_{e}=n_{p}+n_{h}$.

Then, the coupled equations for the densities can be written as

$A_{p p} \frac{d n_{p}}{d x}+A_{p h} \frac{d n_{h}}{d x}=W_{p x}$,

$A_{h p} \frac{d n_{p}}{d x}+A_{h h} \frac{d n_{h}}{d x}=W_{h x}$,

where

$A_{p p}=1-A_{p} A_{e}$

$A_{p h}=-A_{p} A_{e}$

$A_{h p}=-A_{h} A_{e}$

$A_{h h}=1-A_{h} A_{e}$

and

$A_{p}=-\frac{n_{p}^{3}}{U^{2}}$,

$A_{h}=-\frac{n_{h}^{3}}{U^{2} \alpha \mu}$,

$A_{e}=-\frac{1}{2} \frac{\beta_{e}}{n_{e}}$,

$W_{p x}=A_{p}\left[\left(v_{p y}-v_{e y}\right) B_{z}-\left(v_{p z}-v_{e z}\right) B_{y}\right]$

$W_{h x}=A_{h}\left[\left(v_{h y}-v_{e y}\right) B_{z}-\left(v_{h z}-v_{e z}\right) B_{y}\right]$.

It is clear that the coupling between the proton and heavy ion fluids is caused by differential streaming between both ion species, which generates force terms proportional to $\left(\mathbf{v}_{e}-\mathbf{v}_{i}\right) \times \mathbf{B},(i=p, h)$. 

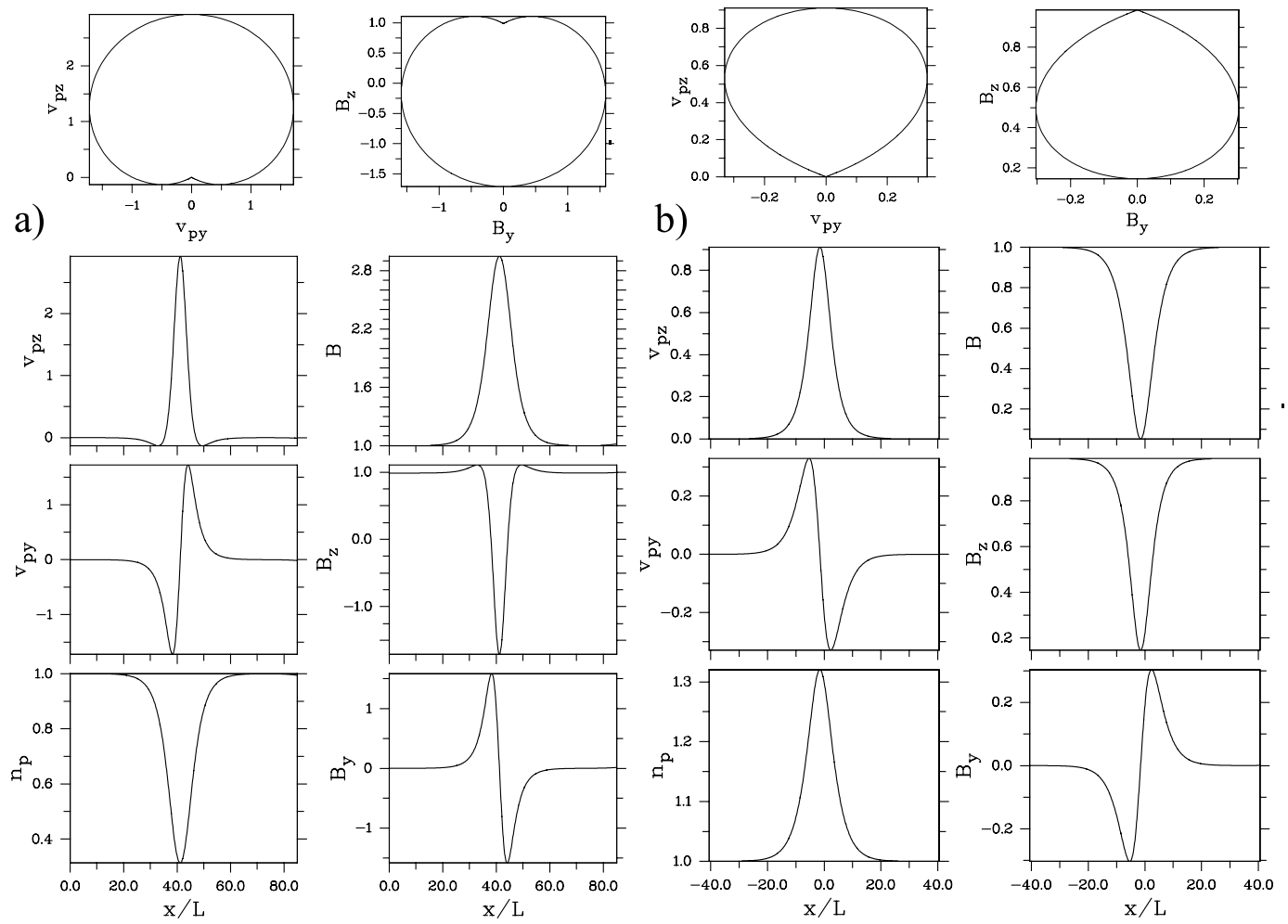

Fig. 2. Structure of "bright" (a) and "dark" (b) Hall-MHD solitons in a proton-electron plasma propagating at $\theta=80^{\circ}$ relative to the magnetic field with $U=0.16$ (the velocity is normalized to the Alfvén speed; $\beta_{e}=3$ ).

The system of coupled, nonlinear differential Eqs. (21), (24), (28) for $v_{p y}, v_{p z}, B_{y}, B_{z}, n_{p}, n_{h}$, along with the algebraic relations (23), (25), (27) for the remaining quantities $v_{h y}, v_{h z}, v_{e y}, v_{e z}, v_{p x}, v_{h x}$ determine the spatial structure of stationary nonlinear waves in a bi-ion plasma. Linearization of these equations in the neighbourhoud of the initial state solutions yields of the evanescent type, $\exp (\kappa x)$, in certain parameter regimes. Before discussing the properties of these stationary solutions, it is useful to consider the behaviour of the propagating bi-ion plasma modes, which is well known from the literature (see e.g. Smith and Bryce, 1964). The addition of a secondary ion population to a hydrogen magnetoplasma generates additional "heavy-ion modes", which also modify the main Hall-MHD modes (e.g. Krauss-Varban et al., 1994; Vocks et al., 1999) through mode splitting effects. In our fluid approach, the dispersion curves of these waves may be obtained from the linearized stationary equations by using the transformation $U \rightarrow \frac{\omega}{k}$.

Figure 3 shows the diagnostic diagram for low-frequency waves propagating at an angle of $\theta=30^{\circ}$ in a cold $\left(\beta_{e}=0\right)$ and warm $\left(\beta_{e}=1\right)$ single-ion and bi-ion plasma $(\alpha=$ $\left.n_{h o} / n_{p o}=0.2, \mu=m_{h} / m_{p}=10\right)$. In the latter case, a new "heavy-ion mode" appears with a cut off frequency above which the waves propagate with the fast mode speed. Consequently, new "gaps" in the $\frac{\omega}{k}-k$ diagram appear in which evanescent waves may exist. The circle in the bottom panel of Fig. 3 marks an interesting feature, namely a throat in the gap between the two wave modes. This has interesting consequences for stationary waves, which we discuss subsequently.

Before analysing these new features we first consider how the properties of single-ion solitons, described in the previous section, are modified by the admixture of a second ion population. Figure 4 shows the dispersion relation of stationary waves, $k=k(U)$, in a bi-ion plasma $(\mu=4)$ for different abundance ratios $\alpha=n_{h o} / n_{p o}$. For very low densities of the heavy ions, evanescent waves $(\operatorname{Im}(k)>0)$ are expected (not too different from the situation in a single-ion plasma) in the gap between the slow and intermediate modes at $U \sim 0.13$. It is worth noting that the real part corresponding to this root is zero. (The curve with $\operatorname{Re}(k) \neq 0$ at $U \sim 0.13$ belongs to "heavy ion mode".) With increasing heavy-ion density, the spatial growth rate $\operatorname{Im}(k)$ decreases, and the gap of permitted soliton velocities $U$ narrows with the implication that the amplitude of the soliton decreases.

Figure 5a shows the structure of a "dark" soliton propagating obliquely to the magnetic field $\left(\theta=80^{\circ}\right)$. The dropout of the magnetic field value in the "magnetic hole" is smaller than in the single-ion case. Another interesting feature is the appearance of small wave oscillations superimposed on the soliton structure. Figure $5 \mathrm{~b}$ shows these periodical waves in more detail. They have $\operatorname{Re}(k) \sim 2$ and belong to the "heavy ion mode" (with $\operatorname{Im}(k)=0$, see Fig. 4. Moreover, Fig. 4 displays a new feature, which is specifically unique to the bi-ion plasma. An additional region of spatially grow- 


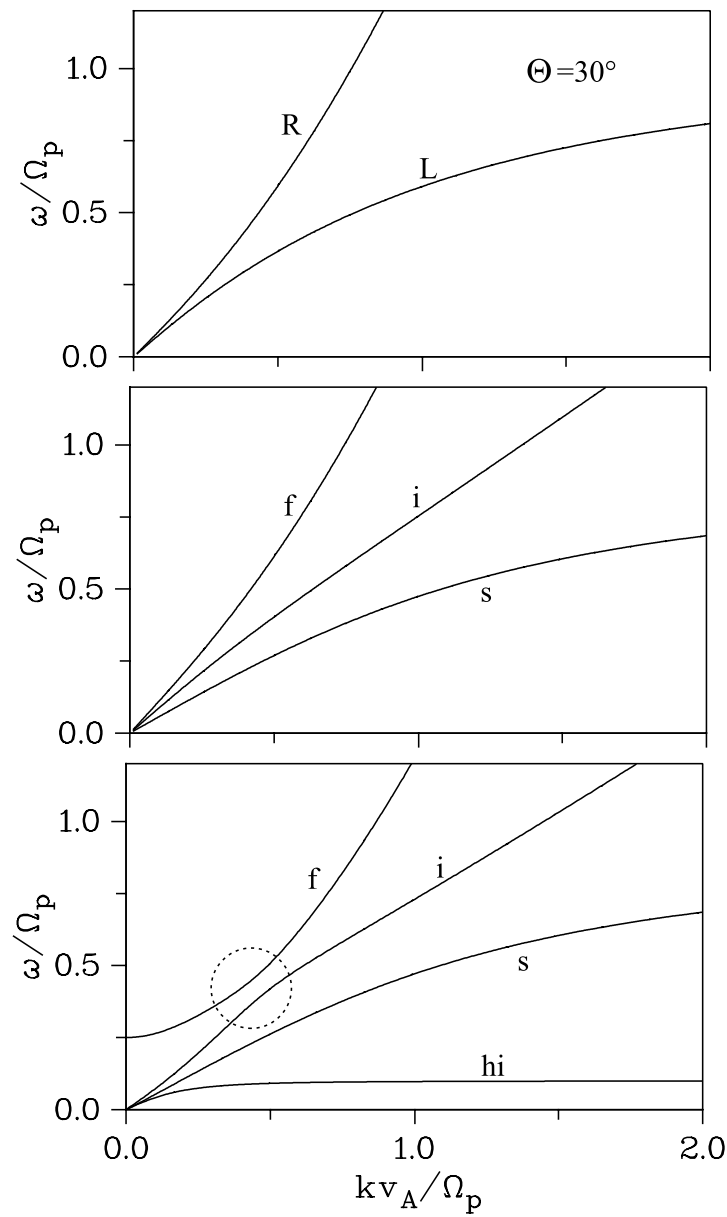

Fig. 3. Dispersion of LF electromagnetic waves in a cold plasma of protons and electrons (top), in a proton-electron plasma with cold protons and warm $\left(\beta_{e}=1\right)$ electrons (middle), and in a biion plasma with an admixture of heavy ions $(\alpha=0.2, \mu=10)$ (bottom). Addition of heavy ion population modifies the characteristics of plasma waves by producing a cutoff frequency and the appearance of a new mode. The circle shows a throat in the gap where evanescent waves may exist. $R, L, f, i, s$ and $h i$ mark the right-hand polarized, the left-hand polarized, the fast, the intermediate, the slow and the heavy-ion modes, respectively.

ing solutions appears at $U \sim 0.23$, in a gap between the intermediate and heavy ion mode which has its cutoff frequency at $\left(k \rightarrow 0\right.$ at $\left.\omega^{*}=\left(n_{p} \Omega_{h}+n_{h} \Omega_{p}\right) / n_{e}\right)$. The growth rate $\operatorname{Im}(k)$ increases with the addition of heavy ions, and the range of soliton speeds $U$ becomes broader. The characteristic new feature of this root is the appearance of a finite value of $\operatorname{Re}(k)$, together with $\operatorname{Im}(k) \neq 0$. That is to say, the roots of the dispersion relation $D(k U, \mathbf{k}=0)$ are complex, which has its manifestation in the "link" between the curves describing different wave modes in the $\operatorname{Re}(k)-U$ diagram. Within this region with $\operatorname{Re}(k) \neq 0, \operatorname{Im}(k) \neq 0$, stationary structures comprise a "normal soliton" modulated by spatial oscillations. These structures have been termed "oscillitons" (Sauer et al., 2001). More details of the dispersion characteristics of stationary waves in a cold bi-ion plasma are given in
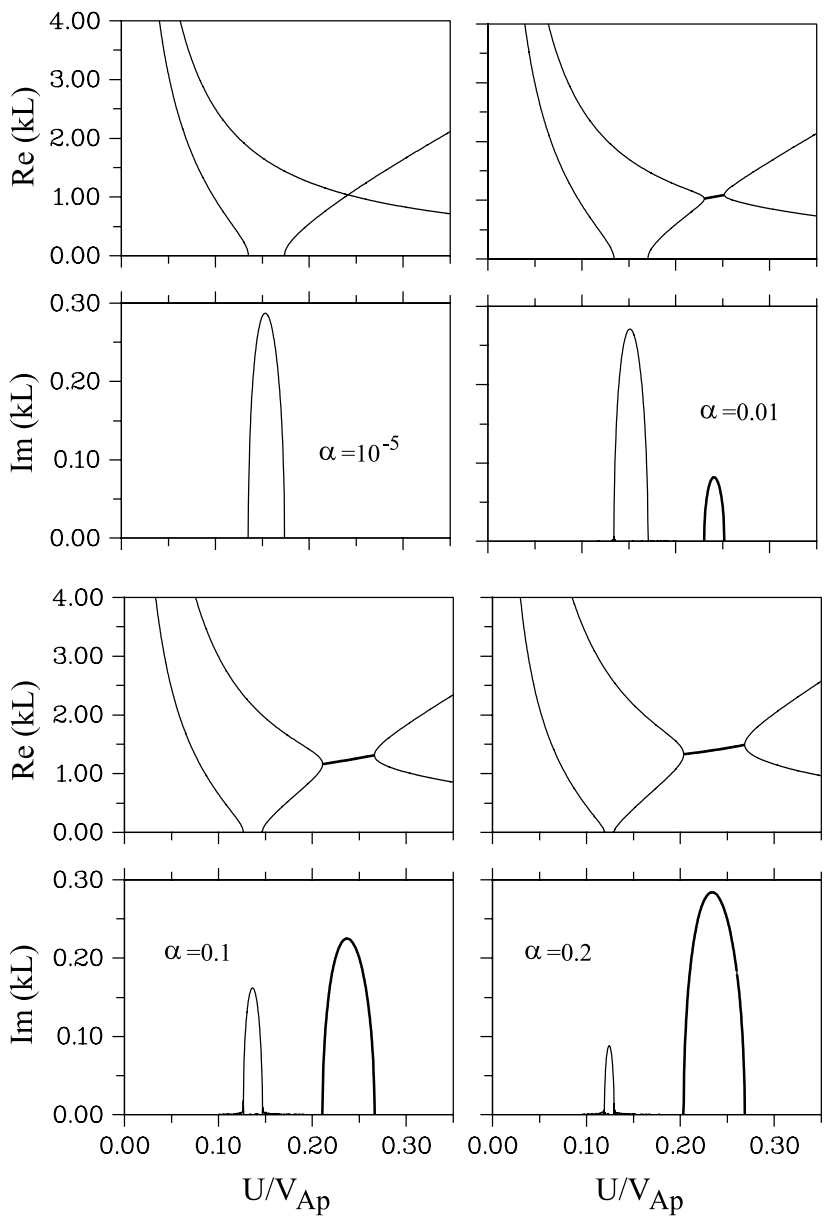

Fig. 4. Roots of the dispersion relation for stationary waves in a bi-ion plasma, $k=k(U)$ for different abundance of heavy ions $\alpha=10^{-5}, 10^{-2}, 10^{-1}$ and $0.2\left(\theta=80^{\circ}, \beta_{e}=3, \mu=4\right)$. The thick solid lines mark the "oscilliton solution".

Appendix 1.

An example of an "oscilliton" obtained by solving numerically the governing system of equations for stationary waves in a bi-ion plasma is shown in Fig. 6a. Several successive structures with an oscillating pattern superimposed on a sechtype soliton appeared. Figure $6 \mathrm{~b}$ shows the fine structure of the solution. An interesting feature is displayed in the plots of the proton velocity $\left(v_{p x}\right)$ and the density $n_{p}$ in the centre of the structure. In the frame moving with the soliton speed $(U)$, this feature is revealed as a "break" in the growing and oscillating behaviour of $v_{p x}$. In the laboratory frame, the heavy ions are decelerated $\left(U-v_{h x}\right)$, as one would expect for a compressive solution. The protons, at first, are also decelerated $\left(\left(U-v_{p x}\right)\right.$. However, near the centre they begin to be accelerated. This effect is intensified as the heavy-ion mass increases. A similar behaviour of both ion fluids, but without oscillations, is also observed in compressive solitons propagating perpendicular to the magnetic field (Sauer et al., 2000; McKenzie et al., 2001; Dubinin et al., 2002) and is caused by the momentum exchange between the protons, the heavy ions and the magnetic field. 

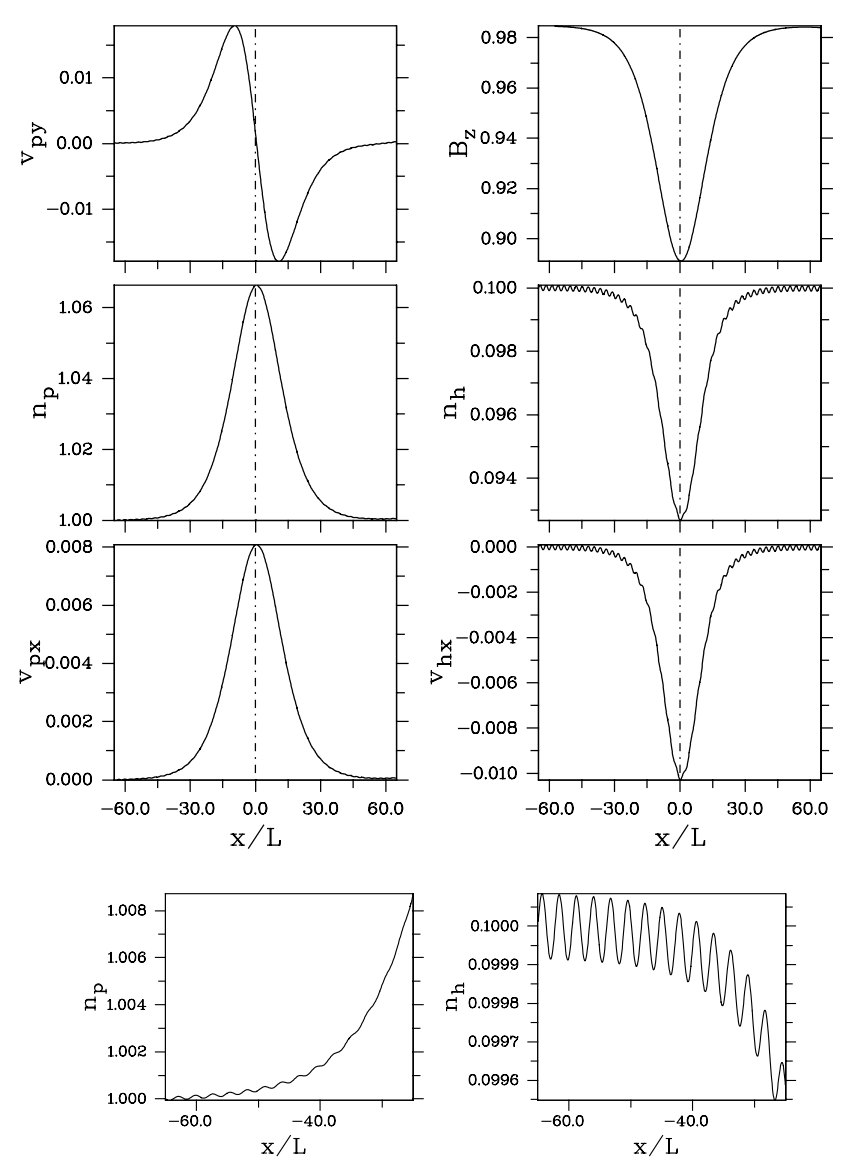

Fig. 5. Structure of a "dark" soliton propagating obliquely to the magnetic field $\left(\theta=80^{\circ}\right)$ in a bi-ion plasma $\left(U=0.13, \beta_{e}=\right.$ $3, \alpha=0.1, \mu=4)$. In comparison to the corresponding singleion soliton (see Fig. 2b) it has weaker magnetic field depression and exhibits small-scale oscillations as manifestation of the heavyion mode which is present in addition to the soliton solution (see Fig. 4).

\section{Discussion}

The addition of a second ion population leads to significant modifications in the structure of stationary waves. So-called "bright" and "dark" solitons in a single-ion plasma (see e.g. Baumgärtel et al., 1997) can exist only in a narrow range of the soliton speeds. This range and the soliton amplitude decrease with increasing abundance of the second ion population. However, an interesting new feature appears in a bi-ion soliton structure which now contains embedded small-wave oscillations. Moreover, a new type of stationary solution exist, which we have called an "oscilliton", to indicate that it has oscillating as well as classical soliton properties. These oscillitons are characterized by a "normal" soliton structure modulated by oscillations. Such strong nonlinear structures arise because of very specific dispersion characteristics. The inclusion of a second ion population results in a splitting of "intersecting" wave modes with the appearance of a gap in the $(\omega / k-k)$ phase space. In "normal" gaps with $\operatorname{Re}(k)=0$, evanescent stationary waves (which describe the initial evo-
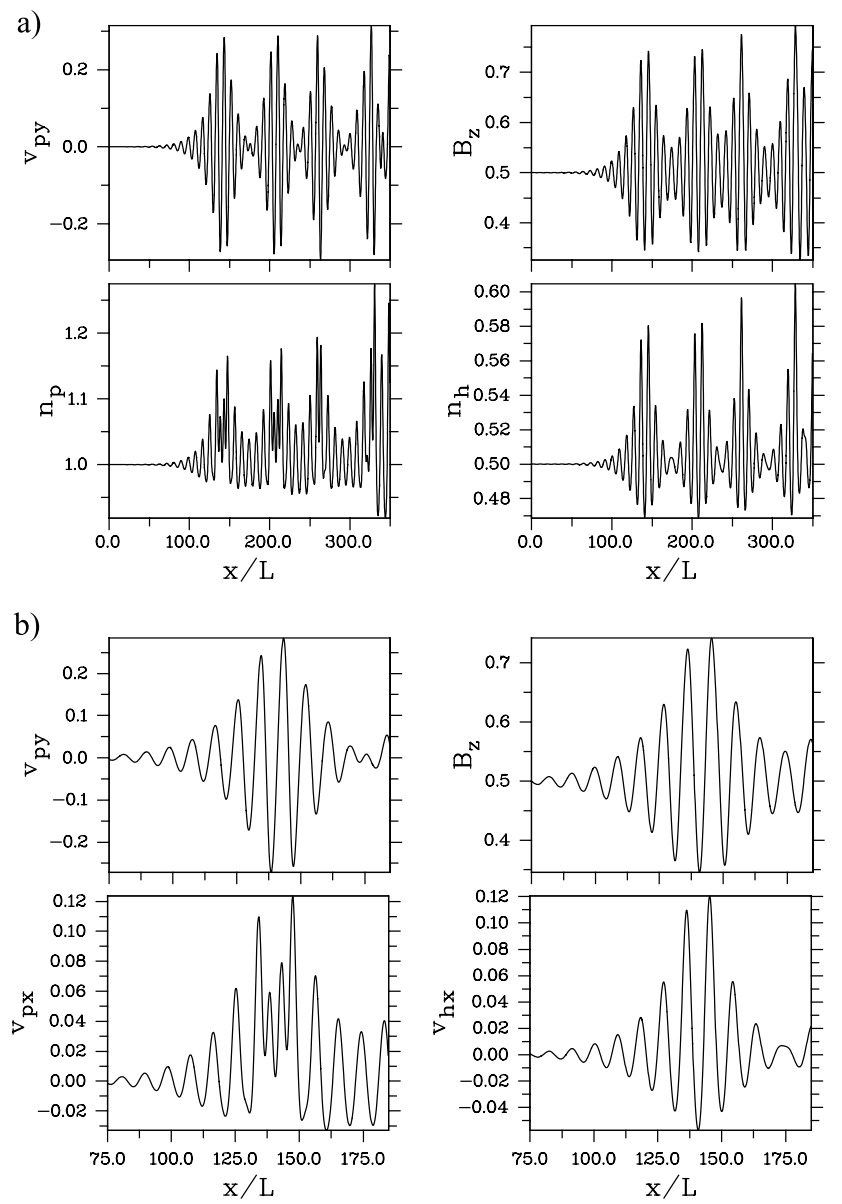

Fig. 6. (a) Example of bi-ion oscillitons propagating at $\theta=$ $30^{\circ},\left(U=0.87, \beta_{e}=0.5\right)$, (b) Fine structure of the oscilliton.

lution of a soliton structure) exist. The roots of the dispersion equation $D(\omega=k U, k)=0$, which belong to these solutions, are purely imaginary $\left(k^{2}<0\right)$. For stationary waves propagating obliquely to the magnetic field in a biion plasma, however, the gap has a throat which modifies the soliton structure in an essential way such that the equation $D(k U, k)=0$ for stationary waves now yields complex roots $k=k_{r}+i k_{i}$. In this case, the initial evolution of a soliton contains an oscillating part superimposed on the exponentially growing or decaying structure.

These oscillitons (Sauer et al., 2001) look like wave packets with a small-scale length determined by $k_{r}=\operatorname{Re}(k)$. The amplitude and width of the oscilliton depend on the interplay between dispersion and nonlinearity, as in the usual solitons. Generally, we may postulate that any plasma system with throat-like dispersion features will possess nonlinear stationary solutions with "oscilliton" structure. For example, it is interesting to note that for a nongyrotropic distribution of the heavy ion population, typical for small comets, the splitting between the fast $(\mathrm{R})$ and intermediate $(\mathrm{L})$ modes gives rise to a throat-shaped gap in the $\omega / k-k$ space, even for the case of parallel wave propagation. In a plasma with a gyrotropic heavy-ion distribution, this splitting disappears at $\theta=0$. In 

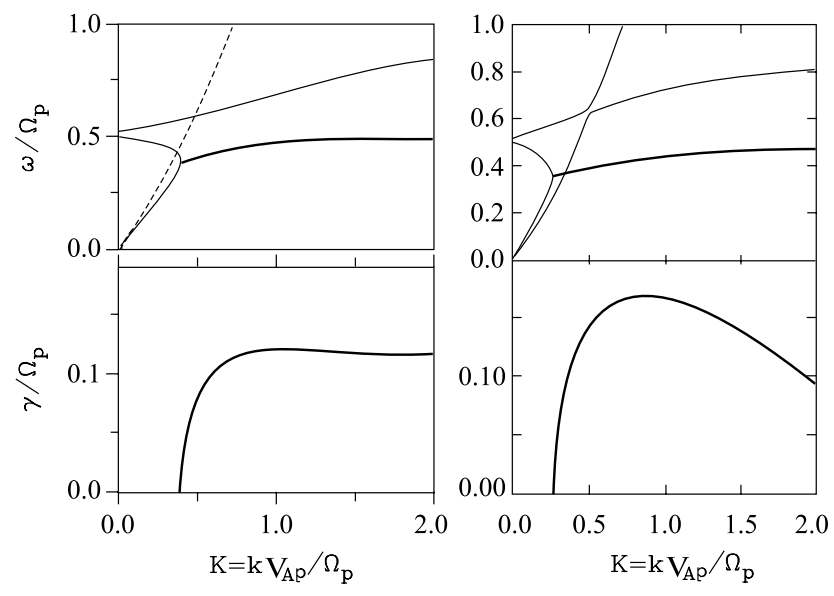

Fig. 7. Dispersion of parallel propagating LF waves in an anisotropic bi-ion plasma. (left) The distribution of the heavy ions (alpha particles with $\alpha=0.05$ ) is gyrotropic with $V_{\perp} / V_{A}=1$. (right) The distribution of alpha-particles is a non-gyrotropic ring (Motschmann et al., 1997).

Fig. 7, the dispersion of low-frequency waves propagating parallel to the magnetic field for these two cases is displayed. The second population consists of alpha particles, $\alpha=0.05$, with thermal anisotropy $\left(T_{\perp} \gg T_{\|}\right)$of the minor ion component. The wave modes of the gyrotropic plasma are shown in the left panels. These represent the right-hand polarized mode (dashed lines), which remains without modification by the second ion population, and the left-hand polarized modes $\left(L_{p}, L_{h}\right)$ with signatures, indicating the coupling between protons and heavy ions. The right panels of Fig. 7 show the dispersion curves for the case that the heavy ions have a nongyrotropic ring distribution (Motschmann et al., 1997). In both cases, the existing temperature anisotropy drives the $L_{h}$ mode to be unstable. It is remarkable that the non-gyrotropy splits the upper branches of the $R$ and $L$ modes. Thus, a gap appears in the $\omega / k-k$ space which may lead to oscillitons. Similar dispersion features are also expected in a (single-ion) plasma consisting of two proton populations with different temperatures (Sauer et al., 2001).

A gap in the phase space $\omega / k-k$ with a characteristic throat feature selects a frame of reference in which both the phase and group velocities become zero. In this reference frame, which is easily transformed to an observer reference frame by selecting an angle of wave propagation $(\omega=k U \cos \phi)$, a stationary pattern of standing waves may arise. In a recent paper by Sauer et al. (2002b) the existence of phase- and group-standing whistler waves was demonstrated and may be related to whistler oscillitons giving rise to coherent wave packets ("lion raors") observed in the Earth's magnetosheath (Baumjohann et al., 1999).

Satellite measurements provide us with many examples in which nonlinear coherent structures with oscillating waveforms are observed. We suggest that such structures may be oscillitons, as seen by an observer at rest, as temporal wave packets with superimposed oscillations. The frequency of the
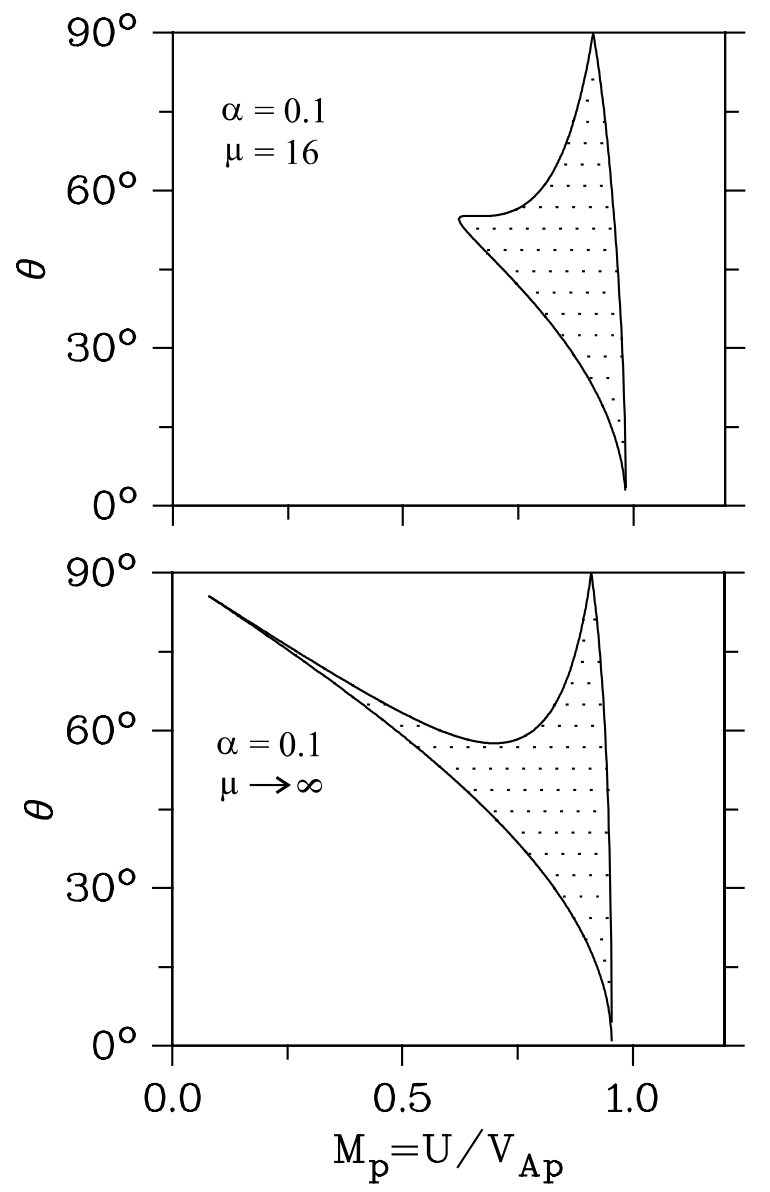

Fig. 8. Parameter space $\left(\theta, M_{p}\right)$ where oscilliton-type solutions exist (dotted area). Top: $\alpha=0.1, \mu=16$; bottom: the same heavyion density $\alpha$, but massive ion approximation, $\mu \rightarrow \infty$. $M_{p}$ is the Mach number based on the proton Alfvén speed.

observed waves is determined roughly by their wave number and the flow velocity, $\omega^{\prime}=k U \cos \phi$. The wave number of stationary waves is $\sim \omega^{*} / V_{A}$, where $\omega^{*}$ is the cutoff frequency, which coincides approximately with the gyrofrequency of the heavy ions, if they constitute a minor ion population $\left(n_{h} \ll n_{p}\right)$. We then have $\omega^{\prime} \approx \frac{V \cos \phi}{V_{A}} \Omega_{h}$. For stationary waves in the laboratory frame $V \cos \phi \sim V_{A}$, the observed frequency occurs close to the gyrofrequency of the heavy ions $\omega^{\prime} \sim \Omega_{h}$.

We list briefly several examples of observations of coherent wave packets with characteristics similar to oscillitons. During the Galileo flyby near Io, the magnetometer recorded bursts of highly-monochromatic waves near the gyrofrequency of $\mathrm{SO}_{2}^{+}$ions (Kivelson et al., 1996). Io is immersed in a plasma flow which is supplied by neutral gases that are lost from Io, and ionized. The dominant ions, $\mathrm{O}^{+}$, $S^{++}, S^{+}$, which are long-lived species, are lost by outward radial transport on a time scale of $\sim 20-100$ days. On the other hand, $\mathrm{SO}_{2}^{+}$ions, which are a minor ion component $(\sim 5 \%)$, quickly dissociate. Therefore, as suggested by Huddleston et al. (1997), the emissions at approximately $\Omega_{\mathrm{SO}_{2}^{+}}$ 
are not strongly suppressed by the background plasma.

The plasma environment near Mars contains ionized hydrogen atoms originating from the extended hydrogen exosphere. These ions are picked up by the solar wind and form a second proton population. Secondary protons can be explicitly included in the Hall-MHD equations with different temperature and density from the main proton population. The dispersion pattern of such a bi-proton plasma also contains a throat feature in the $\omega / k-k$ phase space, which may explain the occurrence of pronounced coherent wave packets in the upstream region of Mars (Mazelle, 2000). In recent papers by Sauer et al. $(2001,2002 a)$ it has been shown that the observed emission at the proton cyclotron frequency may be explained through the formation of oscillitons in a plasma with two proton populations with a relative drift.

Foreshocks near planetary bowshocks provides another possible region where oscilliton structures can be generated by the presence of a second proton population formed by upstream moving solar wind protons reflected at bowshocks.

\section{Appendix A Stationary waves in bi-ion plasma}

In general the dispersion equation for a plasma may be denoted as

$D(\omega, \mathbf{k})=0$

for plane wave disturbances varying as exp $i(\omega t-\mathbf{k r})$. Putting $\omega=k U$ in a frame moving with speed $U$ and solving Eq. (22) for $k$ as a function of $U$ with the angle $\theta$ between $\mathbf{k}$ and the magnetic field $\mathbf{B}$, yields

$k=k_{j}(U, \theta)$,

where $j$ denotes the roots of $D(U k, \mathbf{k})=0$. Soliton-like solutions of the full nonlinear equations, in general, require that in the initial state the stationary waves be of the evanscent type $\left(k_{j}^{2}<0\right)$. Here we show that in bi-ion plasma consisting of protons and heavy ions, there exist regimes in the "Mach number $-\theta$ " space in which $k_{j}^{2}<0$ and also regimes where $k_{j}^{2}$ is complex. This latter case is interesting because it implies that the corresponding soliton structure should exhibit an oscillating spatial structure arising from the $R e\left(k_{j}\right)$ superimposed on the "normal" spatial structure associated with an $\operatorname{Im}\left(k_{j}\right)$.

To illustrate this point, consider a cold bi-ion plasma for which the dispersion relation is (see e.g. Stix, 1994).

$$
\begin{aligned}
& k_{\perp}^{2}=\frac{\left(k_{\|}^{2}-L\right)\left(k_{\|}^{2}-R\right)}{\left(S-k_{\|}^{2}\right)} \\
& R, L=\sum_{i} \frac{\omega^{2} / V_{A i}^{2}}{\left(1 \pm \omega / \Omega_{i}\right)} \\
& S=\sum_{i} \frac{\omega^{2} / V_{A i}^{2}}{\left(1-\omega / \Omega_{i}\right)}=\frac{L+R}{2},
\end{aligned}
$$

where $V_{A i}^{2}=B^{2} / \mu_{o} m_{i} n_{i}, \Omega_{i}=e q_{i} B_{o} / m_{i}$. Electron inertia has been neglected, which is permissible for wave frequencies less than the lower hybrid frequency. Inserting the stationary wave condition $\omega=U k$ into Eq. (A3a) and writing

$\left(k_{\perp}, k_{\|}\right)=k(\sin \theta, \cos \theta)$

we obtain after re-arrangement

$(1-r)(1-l)=\sin ^{2} \theta(1-s)$,

where

$$
\begin{aligned}
& r, l=\sum_{i} M_{i}^{2}\left(1 \pm k / \kappa_{i}\right), \\
& s=\sum_{i} M_{i}^{2}\left(1-k^{2} / \kappa_{i}^{2}\right),
\end{aligned}
$$

where

$M_{i}^{2}=U^{2} / V_{A i}^{2}, \kappa_{i}=\Omega_{i} / U, M^{2}=\sum_{i} M_{i}^{2}$.

For a bi-ion plasma $(i=p, h)$ Eq. (A5a) becomes

$$
\begin{aligned}
& \left(1-M^{2}+\frac{k^{2}}{\kappa_{p} \kappa_{h}}\right)^{2}-b k^{2}= \\
& \quad\left(1-M^{2}+\frac{k^{4}}{\kappa_{p}^{2} \kappa_{h}^{2}}-b_{e} k^{2}\right) \sin ^{2} \theta,
\end{aligned}
$$

in which

$$
\begin{aligned}
& b=\left(\frac{1-M_{h}^{2}}{\kappa_{p}}+\frac{1-M_{p}^{2}}{\kappa_{h}}\right)^{2}-\frac{2\left(1-M^{2}\right)}{\kappa_{p} \kappa_{h}}, \\
& b_{e}=\frac{1-M_{h}^{2}}{\kappa_{p}^{2}}+\frac{1-M_{p}^{2}}{\kappa_{h}^{2}}
\end{aligned}
$$

Equation (A6a) is bi-quadratic in $k^{2}$, i.e.

$A k^{4}-B k^{2}+C=0$

in which

$A=a \cos ^{2} \theta, a=1 /\left(\kappa_{p}^{2} \kappa_{h}^{2}\right)$,

$B=b-\frac{2\left(1-M^{2}\right)}{\kappa_{p} \kappa_{h}}-b_{e} \sin ^{2} \theta$,

$C=\left(1-M^{2}\right)\left(\cos ^{2} \theta-M^{2}\right)$.

Thus, the solutions of Eq. (A7a) may be written as

$k^{2}=\frac{B \pm \sqrt{B^{2}-4 A C}}{2 A}$.

Hence, if $\cos \theta<M$ (implying $C<0$ ), $k_{-}^{2}<0$, yielding evanescent stationary waves which describe the initial evolution of a "normal" soliton. On the other hand, if $B^{2}<4 A C$, Eq. (A8) yields complex $k=k_{r} \pm i k_{i}$. In this case, the initial evolution of a soliton contains an oscillating 
part superimposed on the exponentially growing or decaying structure. The region of parameter space in which this occurs is given by the separation curve $B^{2}=4 A C$. The parameter space $\left(\theta, M_{p}\right)$ where oscilliton-type solutions exist (marked by the dotted area) is depicted in Fig. 8 for two cases: $\alpha=0.1, \mu=16$ and $\alpha=0.1, \mu \rightarrow \infty$ (massive ion approximation). It is interesting to note that for Mach numbers slightly below 1 , oscillitons may exist for nearly parallel and transverse propagation.

Acknowledgements. E. Dubinin thanks the Deutsche Forschungsgemeinschaft and the Max-Planck-Gesellschaft for supporting this work by grants. The work was also supported by INTAS-ESA-9900066 grant.

\section{References}

Baumgärtel, K., Dubinin, E., and Sauer, K.: Story, Solar wind magnetic holes: Signatures of slow mode-type MHD solitons, Adv. Space Res., 20, 69, 1997.

Baumgärtel, K.: Soliton approach to magnetic holes, J. Geophys. Res., 9104, 295, 1999.

Baumjohann, W., Treumann, R. A., Georgescu, E., Haerendel, G., Fornancon, K.-H., and Auster, U.: Waveform and packet structure of lion roars, Ann. Geophysicae, 17, 1528, 1999.

Chappel, C. R., Moore, T. E., and Waite, J. H.: The ionosphere as a fully adequate source of plasma for the Eart's magnetosphere, J. Geophys. Res., 92, 5896, 1987.

Dubinin, E. M., Sauer, K., Baumgärtel, K., and Srivastava, K.: Multiple shocks near Mars, Earth, Planets and Space, 50, 279, 1998.

Dubinin, E. M. and Sauer, K.: The Martian magnetosphere-a laboratory for bi-ion plasma investigations, Astrophysics and Space Sci., 264,, 273, 1999.

Dubinin, E. M., Sauer, K., McKenzie, J. F., and Chanteur, G.: Nonlinear waves and solitons propagating perpendicular to the magnetic field in bi-ion plasma with finite plasma pressure, Nonlinear Proc. Geophys., 9, 2, 87-99, 2002.

Hackenberg, P., Mann, G., and Marsch, E.: Solitons in multi-ion plasmas, J. Plasma Physics, 60, 845, 1998.

Huddleston, D. E., Strangeway, R. J., Warnecke, J., Russell, C. T., Kivelson, M., and Bagenal, F.: Ion cyclotron waves in the Io torus during the Galileo encounter: Warm plasma dispersion analysis, Geophys. Res. Lett., 24, 2143, 1997.

Krauss-Varban, D., Omidi, N., and Quest, K. B.: Mode properties of low-frequency waves: Kinetic theory versus Hall-MHD, J. Geophys. Res., 99, 5987, 1994.

Kivelson, M. G., Khurana, K., Walker, R. J., et al.: Io's interaction with the plasma torus: Galileo Magnetometer report, Science,
274, 396, 1996.

Mazelle, C.: CESR, Toulouse, France, private communication, 2000.

McKenzie, J. F., Sauer, K., and Dubinin, E.: Stationary waves in a bi-ion plasma transverse to the magnetic field, J. Plasma Physics, 65, 197, 2001.

McKenzie, J. F. and Doyle, T. D.: Oblique solitons in a cold magnetized plasma, Physics of Plasma, 8, 4367, 2001a.

McKenzie, J. F. and Doyle, T. D.: The properties of fast and slow oblique solitons in a magnetized plasma, Physics of Plasma, 9, 55, 2001 b.

Motschmann, U., Kafemann, H., and Scholer, M.: Nongyrotropy in magnetoplasmas: Simulation of wave excitation and phase space diffussion, Ann. Geophysicae, 15, 603, 1997.

Sauer, K., Bogdanov, A., and Baumgärtel, K.: Evidence of an ion composition boundary (protonopause) in bi-ion fluid simulations of solar wind mass loading, Geophys. Res. Lett., 21, 2255, 1994.

Sauer, K., Dubinin, E., Baumgärtel, K., and Bogdanov, A.: Bow shock splitting in bi-ion flows, Geophys. Res. Lett., 23, 3643, 1996.

Sauer, K., Dubinin, E., and Baumgärtel, K.: Bi-ion structuring in the magnetosheath of Mars, Theoretical modeling, Adv. Space Res., 20, 137, 1997.

Sauer, K., McKenzie, J. F., and Dubinin, E.: Waves and nonlinear structures in bi-ion plasmas, in: Waves in Dusty, Solar and Space Plasmas, (Eds) Verheest, F., Goossens, M., Hellberg, M. A., and Bharuthram, R., p. American Institute of Physics Conference Proceedings Series, Woodhead, NY, USA, 2000.

Sauer, K., Dubinin, E., and McKenzie, J. F.: New type of soliton in bi-ion plasmas and possible applications, Geophys. Res. Lett., 28, 3589, 2001.

Sauer, K., Dubinin, E., and McKenzie, J. F.: Coherent Waves in Multi-Ion Plasmas, Physica Scripta, T98, 52, 2002a.

Sauer, K., Dubinin, E., and McKenzie, J. F.: Coherent wave emission by whistler oscillitons: application to lion roars, Geophys. Res. Lett., submitted, 2002b.

Smith, R. L. and Brice, N.: Wave propagation in multi-component plasmas, J. Geophys. Res., 69, 5029, 1964.

Stix, T.: Waves in Plasmas, American Institute of Physics, New York, 1992.

Verheest, F.: Nonlinear parallel Alfvén waves in cometary plasmas, Icarus, 86, 273, 1990.

Vocks, C., Motschmann, U., and Glassmeier, K.-H.: A mode filter for plasma waves in the Hall-MHD approximation, Ann. Geophysicae, 17, 712, 1999.

Winterhalter, D., Neugebauer, M., Goldstein, B., et al.: Ulysses field and plasma observations of magnetic holes in the solar wind and their relation to mirror-mode structures, J. Geophys. Res., 99, $23371,1994$. 